\title{
Neutrophil CD64 as a marker of infection in patients admitted to the emergency department with acute respiratory failure
}

This article was published in the following Dove Press journal:

Open Access Emergency Medicine

27 May 2014

Number of times this article has been viewed

\section{Andrea Cortegiani \\ Vincenzo Russotto \\ Francesca Montalto \\ Grazia Foresta \\ Pasquale lozzo \\ Santi Maurizio Raineri \\ Antonino Giarratano}

Department of Biopathology and Medical and Forensic Biotechnologies (DIBIMEF), Section of Anesthesiology, Analgesia, Emergency and Intensive

Care, Policlinico "P Giaccone",

University of Palermo, Palermo, Italy
Correspondence: Andrea Cortegiani Department of Biopathology and Medical and Forensic Biotechnologies (DIBIMEF), Section of Anesthesiology, Analgesia, Emergency and Intensive Care, Policlinico "P Giaccone", University of Palermo, Via del Vespro 129, 90I27 Palermo, Italy Tel +39 09l 6552727

Email andreacortegiani@alice.it
Introduction: Cluster of differentiation 64 (CD64) is expressed on neutrophils during bacterial infections and sepsis. The aim of our study was to assess the CD64 expression in patients admitted to the emergency department (ED) with a triage diagnosis of acute respiratory failure (ARF) and/or dyspnea and to verify a relationship between its value and the presence of infection.

Methods: We assessed neutrophil CD64 expression in peripheral blood of patients admitted to the ED with a diagnosis of ARF and/or dyspnea from September 2012 to April 2013. We measured CD64 index by flow cytometry (Leuko64 ${ }^{\mathrm{TM}} \mathrm{kit}$ ) and classified patients as infected within 12 hours from admission, without an infection within 12 hours but infected within 72 hours from admission, and not infected. The primary outcome was differentiating CD64 values of patients with a diagnosis of infection within 12 hours and 72 hours from admission, from those of patients without a diagnosis of infection. The secondary outcome was verifying a relationship between CD64 values and patients' characteristics, Sequential Organ Failure Assessment score, and intensive care unit admission.

Results: Of 212 patients included in the study, $40.1 \%$ were classified as infected within 12 hours from admission, $20.3 \%$ were without an infection 12 hours after admission but were infected within 72 hours, and 39.6\% were not infected. The median CD64 index was higher in patients with an infection on admission (CD64 index: 3.58) than in those not considered infected (CD64 index: 1.37), $P<0.0001$. Among patients not infected at admission, the CD64 index was higher in those with an infection detected during the following hours of observation (CD64 index: 2.75) than in patients without a diagnosis of infection (CD64 index: 1.28), $P<0.0001$. A CD64 index $>3.65$ showed a sensitivity of $94.6 \%$, a specificity of $86.8 \%$, and an area under the receiver-operating characteristic curve of 0.952 for prediction of intensive care unit admission.

Conclusion: CD64 index could represent a useful diagnostic tool for differential diagnosis of ARF in the ED.

Keywords: acute respiratory failure, CD64, CD64 index, infection

\section{Introduction}

Acute respiratory failure (ARF) is a major cause of admission in an emergency department (ED), and the identification of the underlying cause may be challenging. ${ }^{1,2}$ A timely administration of antibiotics in patients with infection has been advocated as a measure to reduce mortality ${ }^{3-5}$ and as a quality of care indicator. ${ }^{6,7}$ Cluster of differentiation 64 (CD64), is the high-affinity immunoglobulin fragment crystallizable $(\mathrm{Fc})$ receptor $(\mathrm{Fc} \gamma \mathrm{RI})$. It is expressed on cells of myeloid lineage, playing a key role in phagocytosis, clearance of immune complexes, antigen presentation, and cytokine release. ${ }^{8,9}$ On resting neutrophils its expression is negligible, but it gradually increases under stimulation by inflammatory cytokines such as granulocyte colony-stimulating 
factor (G-CSF) and interferon (IFN) $-\gamma{ }^{9}{ }^{9}$ Previous studies indicated that quantitative neutrophil CD64 expression is a sensitive and specific tool for diagnosis of sepsis in adults, ${ }^{10-14}$ neonates, and children, ${ }^{15}$ and others have studied quantitative neutrophil CD64 as an indicator of bacterial infection and sepsis in an ED setting. ${ }^{16,17}$ The aim of our study was to assess the CD64 expression in ED patients admitted with a triage diagnosis of ARF and/or dyspnea and to verify a relationship between its value and the presence of infection. Moreover, we sought to determine if a single dose of CD64 on admission could predict the diagnosis of infection established within 72 hours from admission in those patients not considered infected during the first 12 hours. Finally we wanted to verify a relationship between CD64 among patients with a diagnosis of infection and admission to the intensive care unit (ICU) within 72 hours.

\section{Methods}

We obtained the approval from the ethics committee (Comitato Bioetico Azienda Ospedaliera Universitaria Policlinico P Giaccone) for this study. We conducted a prospective study from September 2012 to April 2013 in the Emergency Department (ED) of an urban, tertiary care, teaching hospital (Paolo Giaccone, Palermo, Italy) with approximately 30,000 ED patient visits per year. We enrolled patients aged 18 years or older who were admitted with a nursing first-contact diagnosis (triage process) of ARF and/or dyspnea. We excluded patients with neutropenia (absolute neutrophil count $<1500$ cells $/ \mu \mathrm{L}$ ) or myeloproliferative or lymphoproliferative disorders, or treated with chemotherapy.

The physicians treated all enrolled patients according to their own clinical judgment. Blood samples for neutrophil CD64 quantification were collected together with those required for first laboratory tests performed before any therapeutic intervention. Laboratory technicians who dosed CD64 expression were blinded to the patients' clinical data. After patients' discharge or death, the researchers reviewed the clinical records of each enrolled patient and collected data on a standardized form. They recorded age, sex, comorbidities (acute coronary syndromes [ACS], chronic obstructive pulmonary disease [COPD], chronic heart failure [CHF]), and antibiotic treatment, and they calculated the Sequential Organ Failure Assessment (SOFA) score with available data (worst findings) within 12 hours from ED admission. The researchers classified patients as infected or not infected within 12 hours and 72 hours from ED admission. For this purpose, they registered the final diagnosis of physicians in charge and analyzed clinical signs, laboratory data, and radiological findings relative to the first 12 hours from ED admission and daily up to 72 hours.

During the retrospective analysis, in order to limit elements of subjectivity, they followed the criteria for diagnosing infection in an acute care-setting provided by the US Centers for Disease Control and Prevention/National Health Care Safety network. ${ }^{18}$ When a discrepancy was encountered between the diagnosis made by physicians who treated the patient and researchers' classification or among researchers' judgment, a final decision was made by review of clinical data, discussion and consensus. The same process was adopted in order to solve any doubtful case. The researchers were blind to CD64 value results during the whole clinical data collection, and CD64 values became available only after the complete clinical record review and classification of each enrolled patient.

Primary outcomes were differentiating CD64 values of patients with a diagnosis of infection within 12 hours and 72 hours from admission from those of patients without a diagnosis of infection. The secondary outcome was verifying a relationship between CD64 values and patients' characteristics, SOFA score, and ICU admission. We adhered to the Strengthening the Reporting of Observational Studies in Epidemiology (STROBE) guidelines for conducting and reporting this observational study. ${ }^{19}$

\section{Neutrophil CD64 index quantification}

CD64 levels were studied through a flow cytometric analysis; results were expressed as the standardized CD64 index and collected into a database for later analysis. Ethylenediaminetetraacetic acid samples were processed as soon as available or refrigerated $\left(2^{\circ} \mathrm{C}-8^{\circ} \mathrm{C}\right)$ according to logistic organization. Neutrophil CD64 expression was measured using the Leuko64TM kit (Trillium Diagnostics, LLC, Brewer, ME, USA) and CD64 index was calculated using QuantiCALC ${ }^{\text {TM }}$ software (Trillium Diagnostics, LLC). The CD64 index is obtained from the ratio of the mean fluorescent intensity of the cell population to that of calibrated, fluorescent beads used for standardization and included in the kit. ${ }^{20}$ Flow cytometry was performed by the Coulter ${ }^{\circledR}$ Epic XLтм flow cytometer (Beckman Coulter, Inc., Miami, FL, USA). According to manufacturer's instructions, ${ }^{20}$ an internal negative control (lymphocyte CD64 index $<1$ ) and an internal positive control (monocyte CD64 index $>3$ ) were used to validate each sample.

\section{Statistical analysis}

Variable distribution was analyzed by D'Agostino-Pearson's test. Variables without a normal distribution were expressed 
as median and interquartile ranges (IQR; 25th-75th) and comparisons were performed through Mann-Whitney test. Spearman's rank correlation coefficient (rho) was used to evaluate the correlation between variables. Subgroup analysis was performed using the Kruskall-Wallis test for continuous variables. A frequency table was constructed and the chi-square test was adopted for comparison of more than two proportions. Fisher's exact test was adopted for comparison of two proportions. Receiver-operating characteristic (ROC) curves were created for the CD64 index and its sensitivity, specificity, positive predictive value, and negative predictive value, along with their $95 \%$ confidence intervals (CI), were calculated. The best CD64 cut-off values for infection within 72 hours and ICU admission were calculated. The area under the ROC curve (AUC) for both variables were also determined.

A value of $P<0.05$ was considered statistically significant. Statistical analysis was performed using MedCalc for Windows, version 9.5.0.0 (MedCalc statistical software; Mariakerke, Belgium).

\section{Results}

The flowchart of eligible and enrolled patients is shown in Figure 1. The characteristics of the 212 study patients are summarized in Table 1.
Of 212 patients included in the study, 85 (40.1\%) had signs of infection within 12 hours from ED admission. Of these, 12 had an extrapulmonary source of infection (six urinary tract infections, four abdominal infections, and two soft tissue infections). In 127 patients (59.9\%), a diagnosis of infection was not made within 12 hours from admission. However, in 43 patients (20.3\%) an infection was observed within 72 hours from admission. Among these, eight patients had an extrapulmonary source of infection (five urinary tract infections and three abdominal infections). Treatment included antibiotics in seven patients (16.3\%) not considered infected on admission but meeting criteria for infection within 72 hours. Antibiotics were used in nine patients (10.7\%) who did not meet criteria for infection during the period of observation. These last two groups were not different according to the proportion of antibiotic use $(P=0.40)$. Associated diagnoses in patients without clinical signs of infection are described in Table 2.

The median CD64 index value was 3.58 (IQR 3.00-4.55) in patients with a diagnosis of infection within 12 hours from ED admission and 1.37 (IQR 1.19-2.35) in patients without a diagnosis of infection within 12 hours $(P<0.0001$; effect size, $r=0.69$ ) (Figure 2). A cutoff of 2.79 for the CD64 index showed $82.3 \%$ sensitivity, $88.2 \%$ specificity, and an AUC of

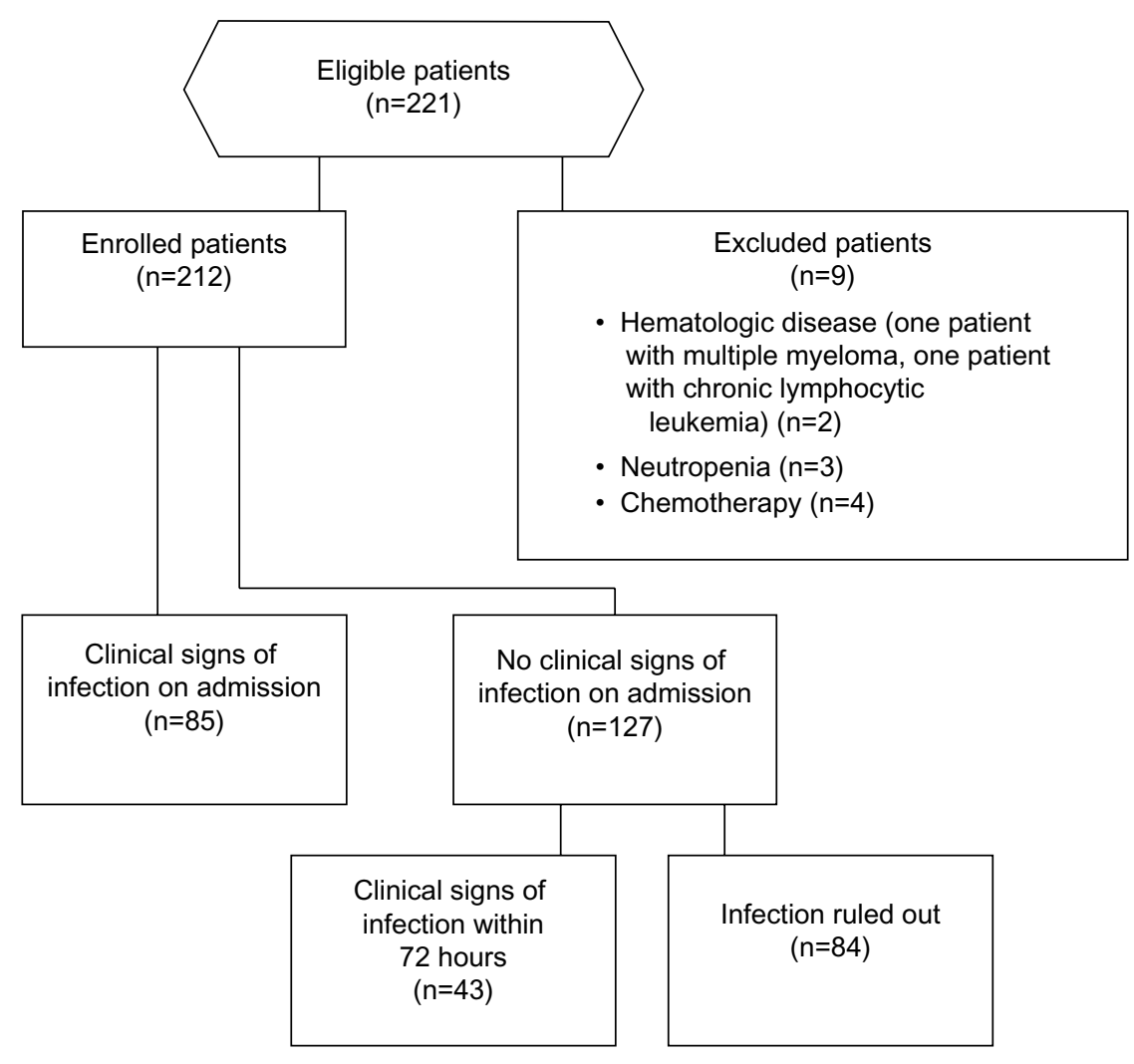

Figure I Study flow chart. 
Table I Clinical characteristics of enrolled patients relative to time from admission in the emergency department

\begin{tabular}{|c|c|c|c|c|c|}
\hline $\begin{array}{l}\text { Patient } \\
\text { characteristics }\end{array}$ & $\begin{array}{l}\text { Signs of infection } \\
\text { within } 12 \text { hours } \\
(n=85) \text { (A) }\end{array}$ & $\begin{array}{l}\text { Signs of infection } \\
\text { within } 72 \text { hours } \\
(n=43)(B)\end{array}$ & $\begin{array}{l}\text { No signs of infection } \\
\text { within } 72 \text { hours } \\
(n=84) \text { (C) }\end{array}$ & $P$-value & $\begin{array}{l}\text { All patients } \\
(n=2 \mid 2)\end{array}$ \\
\hline Age (years, range) & $65.0(59.0-71.0)$ & $66.0(60.0-72.7)$ & $62.0(51.0-71.0)$ & 0.122 & 65.1 \\
\hline \multirow[t]{2}{*}{ Sex, n (\%) } & $M=43(50.6)$ & $M=6 I(47.6)$ & $M=47(55.9)$ & 0.322 & $M=108(50.9)$ \\
\hline & $\mathrm{F}=42(49.4)$ & $\mathrm{F}=67(52.4)$ & $\mathrm{F}=37(44 . \mathrm{I})$ & & $\mathrm{F}=104(49.1)$ \\
\hline SOFA & $3(1-5)$ & $I(I-2)$ & $I(I-2)$ & $<0.000 I^{*}$ & 2 \\
\hline \multicolumn{6}{|l|}{ Comorbidities (\%) } \\
\hline History of ACS & $18(2 \mid .2)$ & $5(I I .6)$ & $39(46.4)$ & $<0.001$ & $62(29.2)$ \\
\hline History of COPD & $61(71.8)$ & $31(72.1)$ & $13(15.5)$ & $<0.0001$ & $105(49.5)$ \\
\hline History of $\mathrm{CHF}$ & $8(9.4 I)$ & $4(9.30)$ & $24(28.6)$ & 0.0013 & $36(17.0)$ \\
\hline WBC count $\left(\times 10^{6}\right.$ cells/L) & $17,400(14,300-23,500)$ & $13,200(12,300-14,900)$ & $11,670(9,115-16,555)$ & $<0.00$ I* & 14,200 \\
\hline Neutrophils $\left(\times 10^{6}\right.$ cells $\left./ \mathrm{L}\right)$ & $\mid 4,200(\mid 1,600-19,050)$ & $10,700(10,100-12,175)$ & $9,360(7,190-13,455)$ & $<0.001^{*}$ & 11,600 \\
\hline
\end{tabular}

Notes: Chi-square test was used for comparison of proportions. Kruskall-Wallis test was used for comparison of continuous variables. $* A$ different from $B$ and $C$.

Abbreviations: A, patients infected within 12 hours from admission; B, patients infected within 72 hours from admission; C, patients not infected; M, male; F, female; SOFA, sequential organ failure assessment; ACS, acute coronary syndrome; COPD, chronic obstructive pulmonary disease; CHF, chronic heart failure; WBC, white blood cell.

0.933 for prediction of infection within 12 hours from ED admission (Figure 3). Among patients without a diagnosis of infection on admission, the median CD64 index of 2.75 (IQR 2.34-3.00) was significantly higher in those who met criteria for infection between 12 hours and 72 hours from admission than in those with a ruled out diagnosis of infection (CD64 index: 1.28, IQR 1.12-1.37, $P<0.0001, r=0.81$ ) (Figure 4). A CD64 index $>1.73$ showed 99\% sensitivity, $96.4 \%$ specificity, a positive predictive value of $93.5 \%(95 \%$ $\mathrm{CI}=82.1 \%-98.6 \%$ ), a negative predictive value of $100.0 \%$ $(95 \% \mathrm{CI}=95.5 \%-100.0 \%)$ and an AUC of 98.9 for prediction of infection during the 72 hours of postadmission observation in patients not considered infected within 12 hours from admission. The CD64 index showed a weak correlation with age ( $r=0.242, P=0.0004)$ and a moderate correlation ${ }^{21}$ with white blood cell count $(r=0.639, P<0.0001)$, neutrophil count $(r=0.637, P<0.0001)$, and SOFA score $(r=0.534$, $P<0.0001$ ) (Figure 5). Patients admitted to the ICU within

Table 2 Associated diagnoses in patients without clinical signs of infection within 72 hours from admission in the emergency department

\begin{tabular}{ll}
\hline $\begin{array}{l}\text { Associated diagnosis } \\
\text { with respiratory failure }\end{array}$ & $\begin{array}{l}\text { No clinical signs of infection } \\
\text { within } 72 \text { hours from } \\
\text { admission }(\mathrm{n}=\mathbf{8 4}, \mathbf{3 9 . 6} \%)\end{array}$ \\
\hline Pulmonary edema & $\mathrm{n}=53,63.1 \%$ \\
Pulmonary embolism & $\mathrm{n}=12,14.3 \%$ \\
COPD & $\mathrm{n}=6,7.14 \%$ \\
Severe asthma & $\mathrm{n}=4,4.76 \%$ \\
Drug overdose & $\mathrm{n}=3,3.57 \%$ \\
Pneumothorax & $\mathrm{n}=2,2.38 \%$ \\
Trauma & $\mathrm{n}=2,2.38 \%$ \\
Inhalation & $\mathrm{n}=2,2.38 \%$ \\
\hline
\end{tabular}

Abbreviation: COPD, chronic obstructive pulmonary disease.
72 hours from admission showed a significantly higher CD64 index (CD64 index: 4.55, IQR 4.11-5.07) than those not admitted to the ICU within the observation interval (CD64 index: 2.92, IQR 2.52-3.38, $P<0.0001)$. The ROC curve of CD64 index for prediction of ICU admission within 72 hours from the first ED observation is shown in Figure 6. A CD64 index $\geq 3.65$ was predictive of ICU admission within 72 hours, with a sensitivity of $94.6 \%$, a specificity of $86.8 \%$, and an AUC of 0.952.

\section{Discussion}

In recent years, several biomarkers were evaluated as diagnostic tools and for prognostic purposes in patients with infection and sepsis in ED and ICU settings. In an ED, an ideal biomarker would improve the initial diagnostic assessment of patients presenting with an infection and thus lead to timely institution of antibiotic therapy, an appropriate duration of

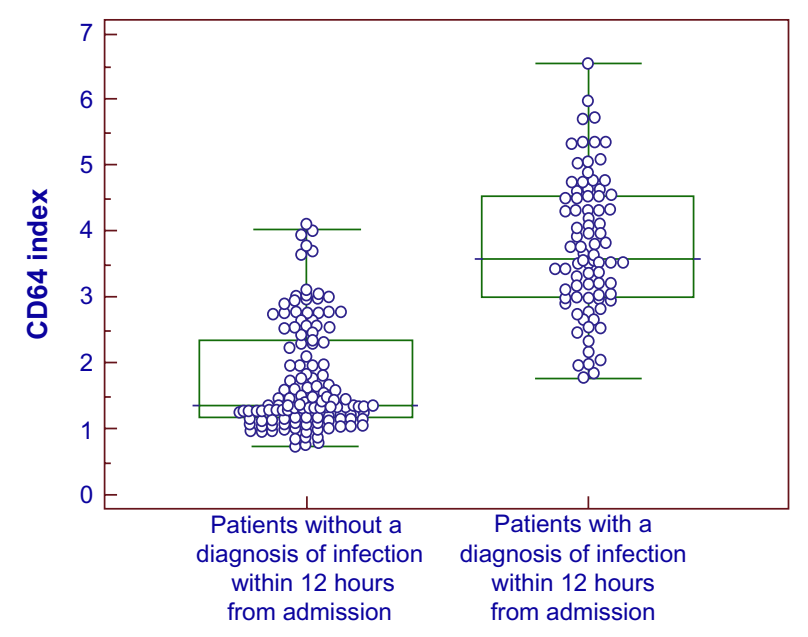

Figure 2 Boxplot distribution of the CD64 index in noninfected and infected patients within 12 hours from admission in the emergency department. Abbreviation: CD64, cluster of differentiation 64. 


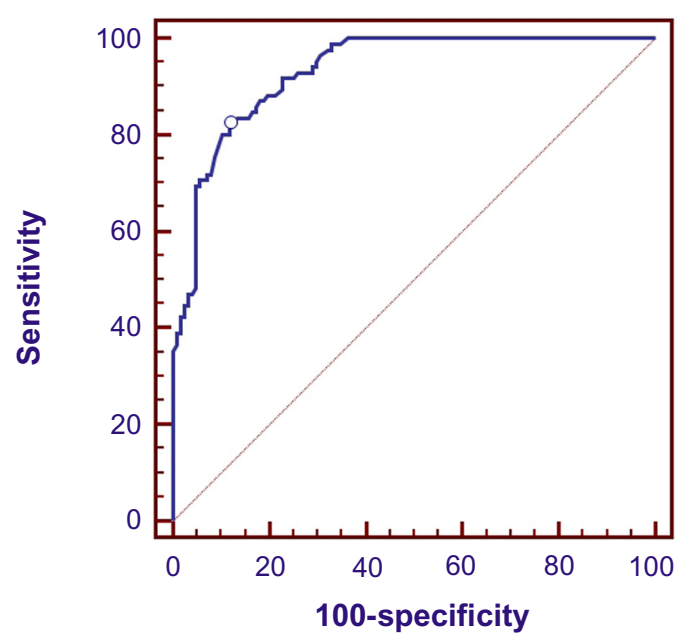

\begin{tabular}{cccccc}
\hline $\begin{array}{c}\text { CD64 index } \\
\text { (Best cut-off) }\end{array}$ & $\begin{array}{c}\text { Sensitivity } \\
(95 \% \mathrm{Cl})\end{array}$ & $\begin{array}{c}\text { Specificity } \\
(95 \% \mathrm{Cl})\end{array}$ & $\begin{array}{c}+\mathrm{PV} \\
(95 \% \mathrm{Cl})\end{array}$ & $\begin{array}{c}-\mathrm{PV} \\
(95 \% \mathrm{Cl})\end{array}$ & $\begin{array}{c}\text { AUC } \\
(95 \% \mathrm{Cl})\end{array}$ \\
\hline 2.79 & 82.3 & 88.2 & 82.4 & 88.2 & 0.933 \\
& $(72.6-89.8)$ & $(81.3-93.2)$ & $(72.6-89.8)$ & $(81.3-93.2)$ & $(0.89-0.96)$ \\
\hline
\end{tabular}

Figure 3 Receiver-operating characteristic curve of CD64 index for prediction of infection within 12 hours from admission in the emergency department. Abbreviations: CD64, cluster of differentiation 64; +PV, positive predictive value; $-\mathrm{PV}$, negative predictive value; AUC, area under the receiver-operating characteristic curve; $\mathrm{Cl}$, confidence interval.

therapy, and the identification of patients with the most severe conditions requiring intensive care. ${ }^{12,13,15,17,22}$ Neutrophil CD64 expression was studied in adults, ${ }^{10-14}$ infants, and children ${ }^{15}$ as an early marker of bacterial infection and sepsis. Its negligible expression in healthy subjects, its prompt upregulation under inflammatory cytokine stimulation, ${ }^{16}$ and its biochemical stability at room temperature for more than 30 hours ${ }^{23}$ represent favorable properties as a marker in an ED setting.

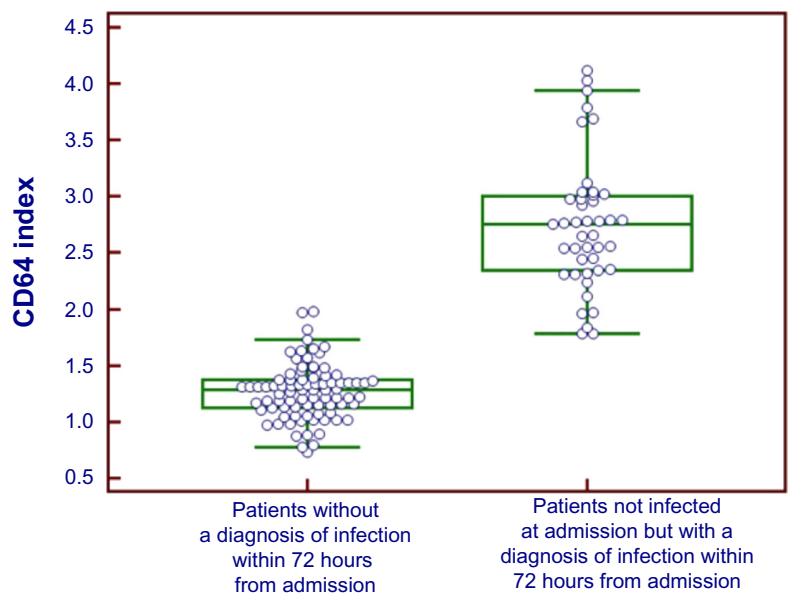

Figure 4 Boxplot distribution of the CD64 index in patients without a diagnosis of infection within 72 hours from admission in the emergency department and in patients without an infection at admission who met criteria for infection within the 72-hour observation period

Abbreviation: CD64, cluster of differentiation 64 .
Moreover, neutrophil CD64 expression seems to be useful in distinguishing systemic infections from other inflammatory conditions. ${ }^{11,24} \mathrm{~A}$ meta-analysis of published studies involving patients of all age groups ${ }^{12}$ revealed a pooled sensitivity of $79 \%$, a specificity of $91 \%$, and an AUC of 0.94 for neutrophil CD64 as a marker of bacterial infection. However, a subgroup analysis revealed a higher performance in the adult population (a mean sensitivity of $90 \%$, a mean specificity of $95 \%$, and an AUC of 0.97 ). Our study suggests a similar high diagnostic accuracy of the CD64 index when adopted as a marker of infection in patients admitted to the ED with a diagnosis of ARF (a sensitivity of $82.3 \%$, a specificity of $88.2 \%$, and an AUC of 0.933 for prediction of infection within 12 hours from ED admission).

It is difficult to compare our results to those of previous studies, as in most of them neutrophil CD64 expression was studied with regard of its ability to predict a culture-proven infection. As a result, optimal diagnostic cutoffs were variable and may also have depended on time of presentation, severity of clinical condition, pathogen characteristics, and antibiotic therapy before admission. ${ }^{25}$ Gámez-Díaz et al, ${ }^{17}$ in a study including septic patients recently admitted to the ED, reported a sensitivity of $65.8 \%$ and a specificity of $64.6 \%$ for CD64 as predictive marker of infection defined by an experts' consensus diagnosis. In the population studied, 22\% of patients were affected by community-acquired pneumonia and the median CD64 was 2.1 MESF (molecules of equivalent soluble fluorochrome) units in this group. Interestingly, a higher CD64 index was measured among patients who met criteria for infection during the 72-hour period of observation but who were not considered infected during the initial evaluation. Compared with white blood cell count, neutrophil count, and SOFA score, the CD64 index was revealed to be a good predictor of infection and could be a useful tool in order to differentiate patients with an infection detected during subsequent hours of observation from those without an infection. Although Davis et al ${ }^{16}$ showed a weak correlation between CD64 and neutrophil count, we agree with their proposed explanation, namely the different specificity for detection of an acute inflammatory response or a different kinetic during the dynamic process of inflammatory response to infection.

Our decision to limit the period of observation to 72 hours from ED admission to follow-up the clinical evolution (a new diagnosis of infection or ICU admission) arose from the need to avoid confounding factors such as hospital-acquired infection and ventilator-associated pneumonia (in those patients admitted to ICU after endotracheal intubation). ${ }^{26,27}$ 
A

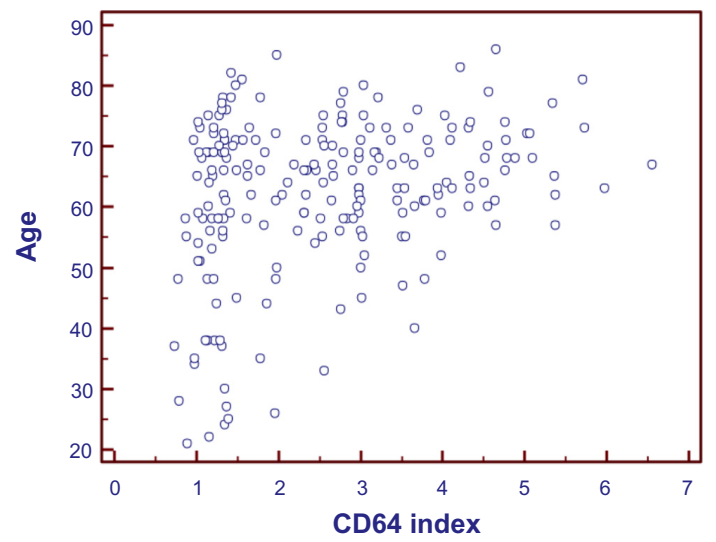

C

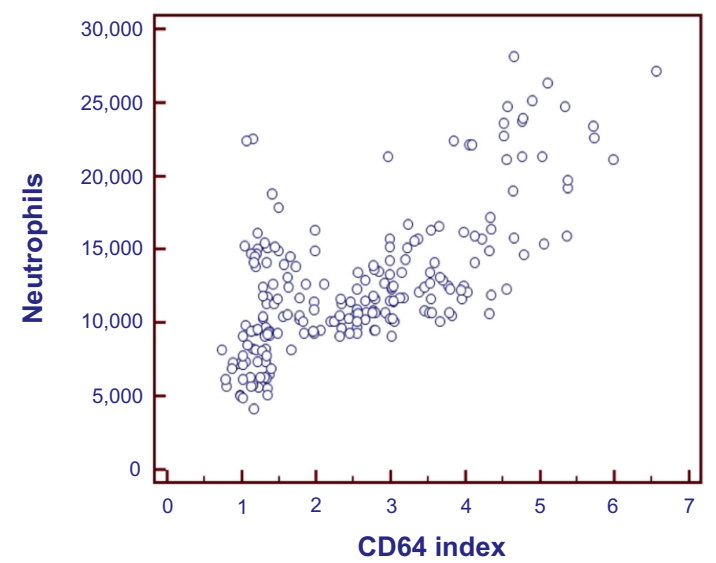

B

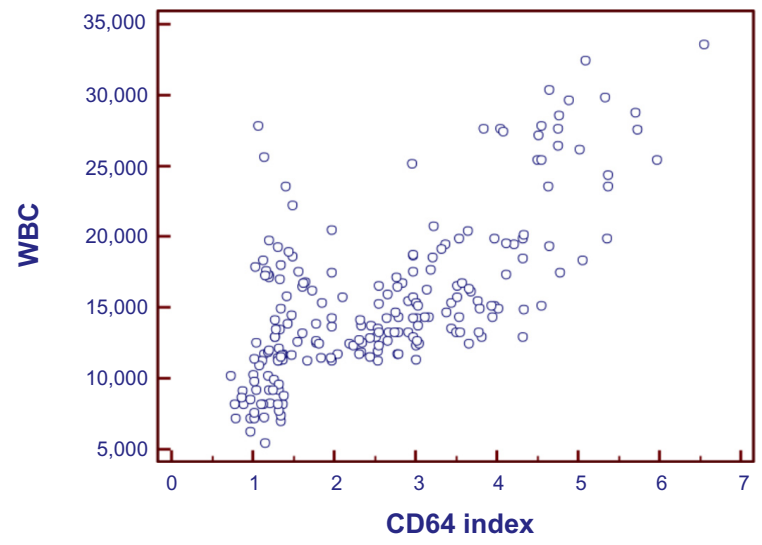

D

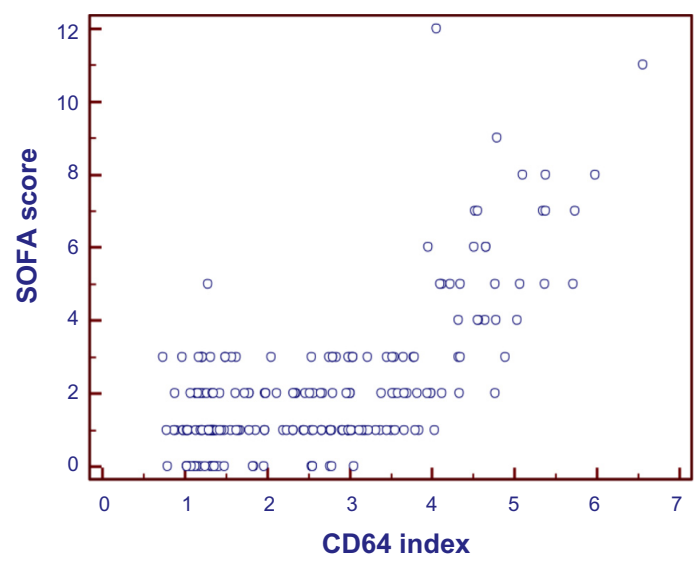

Figure 5 Spearman's rank correlation coefficient between CD64 index and age (A), white blood count $\left(\times 10^{6}\right.$ cells/L) (B), neutrophil count $\left(\times 10^{6}\right.$ cells/L) $(\mathbf{C})$, and SOFA score $(\mathbf{D})$. Abbreviations: CD64, cluster of differentiation 64; WBC, white blood cell count; SOFA, Sequential Organ Failure Assessment.

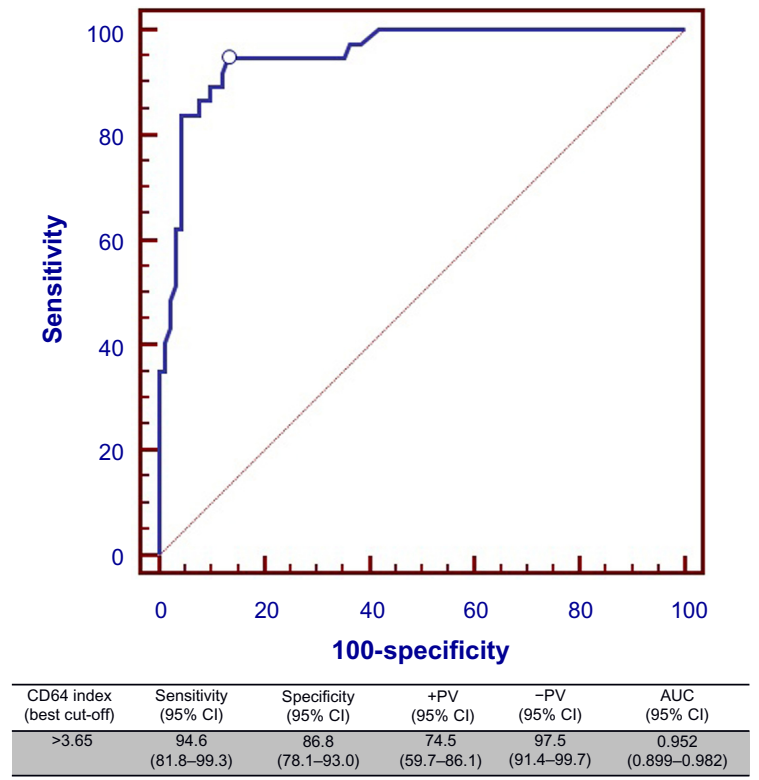

Figure 6 Receiver-operating characteristic curve of CD64 index for prediction of intensive care unit admission within 72 hours from admission in the emergency department.

Abbreviations: CD64, cluster of differentiation 64; +PV, positive predictive value; $-\mathrm{PV}$, negative predictive value; AUC, area under the receiver-operating characteristic curve; $\mathrm{Cl}$, confidence interval.
History of COPD was present in $72.1 \%$ of patients with signs of infection observed within 72 hours from admission. One of the treatments of exacerbations of COPD is corticosteroids. ${ }^{28}$ However, in our study, blood specimens for CD64 analysis were obtained before any therapeutic intervention. Of note, it has previously reported that corticosteroids may not have an influence on CD64 expression by neutrophils. ${ }^{24}$ Patients with severe sepsis or septic shock exhibit a higher level of neutrophil CD64 expression compared to those with a lesssevere infection. ${ }^{29}$

Moreover, a prognostic value for the CD64 index has been recently detected. ${ }^{30}$ We chose ICU admission as secondary outcome since it is a more practical indicator of the severity of illness. In our study, CD64 index showed a high sensitivity (94.6\%) and specificity (86.8\%) for ICU admission within 72 hours from ED admission. However, ICU admission decision, through guided by an institutional protocol, comes from a complete assessment of clinical condition (ie, comorbidities and their severities), and this result should be considered in light of this multifactorial scenario. To date, our study represents the first attempt to evaluate the performance of 
CD64 index not solely as predictor of positive cultures in a population with bacterial infection and/or sepsis, but also for an operational use in detection of patients with infection in a daily ED setting.

Emergency physicians deal with elderly patients with various comorbidities ${ }^{2}$ and are asked for a timely diagnosis and institution of therapy. ${ }^{3}$ Our selected patients, therefore, were representative of the general population of patients admitted to our ED. The adoption of Centers for Disease Control and Prevention/National Health Care Safety Network criteria for diagnosing infections and the discussion of all doubtful cases by the team in order to meet a final classification of patients by consensus represents our attempt to limit possible elements of subjectivity. Although these criteria have been formulated for diagnosis of health care-associated infections, they consist of clinical, laboratory, and radiological findings that commonly characterize patients presenting to the ED with infections.

A limitation of the study is the unique determination of the CD64 index due to economic reasons. In our opinion, this could not be enough to represent the complex dynamic process of inflammation and its evolution after therapy institution. Nevertheless, a punctual neutrophil CD64 quantification was revealed to be a useful tool, especially for diagnosis of infection in those patients without clinical signs on admission. Another limitation is the lack of comparison of the CD64 index with a validated marker of infection. Procalcitonin is a marker of bacterial infection ${ }^{31}$ and it has been studied as a tool in algorithms for diagnosis and prognosis of community-acquired pneumonia ${ }^{25}$ and as a guide for antibiotic therapy in the emergency setting. ${ }^{32}$ However, because this study represents the first attempt to evaluate the diagnostic performance of the CD64 index in the general population admitted to the ED, further studies are needed to confirm our results, evaluate the cost-effectiveness of this marker, and eventually compare its diagnostic performance with other markers of infection.

\section{Conclusion}

According to our study results, the CD64 index seems able to differentiate patients with ARF and/or dyspnea and with a clinically evident infection from those not considered infected within 12 hours and 72 hours from ED admission. Moreover, it seems to identify those patients requiring intensive care within 72 hours. Neutrophil CD64 index could represent a useful diagnostic tool for the differential diagnosis of these patients in the ED.

\section{Acknowledgment}

We thank Professor Simonetta Genova for her contribution to English-language editing of our manuscript.

\section{Disclosure}

The authors report no conflicts of interest in this work.

\section{References}

1. Nee PA, Al-Jubouri MA, Gray AJ, O’Donnell C, Strong D. Critical care in the emergency department: acute respiratory failure. Emerg Med J. 2011;28(2):94-97.

2. Ray P, Birolleau S, Lefort Y, et al. Acute respiratory failure in the elderly: etiology, emergency diagnosis and prognosis. Crit Care. 2006; 10(3):R82.

3. Houck PM, Bratzler DW, Nsa W, Ma A, Bartlett JG. Timing of antibiotic administration and outcomes for Medicare patients hospitalized with community-acquired pneumonia. Arch Intern Med. 2004;164(6): 637-644.

4. Gaieski DF, Mikkelsen ME, Band RA, et al. Impact of time to antibiotics on survival in patients with severe sepsis or septic shock in whom early goal-directed therapy was initiated in the emergency department. Crit Care Med. 2010;38(4):1045-1053.

5. Kumar A, Roberts D, Wood KE, et al. Duration of hypotension before initiation of effective antimicrobial therapy is the critical determinant of survival in human septic shock. Crit Care Med. 2006;34(6): 1589-1596.

6. Pines JM, Morton MJ, Datner EM, Hollander JE. Systematic delays in antibiotic administration in the emergency department for adult patients admitted with pneumonia. Acad Emerg Med. 2006;13(9): 939-945.

7. Mandell LA, Wunderink RG, Anzueto A, et al; Infectious Diseases Society of America; American Thoracic Society. Infectious Diseases Society of America/American Thoracic Society consensus guidelines on the management of community-acquired pneumonia in adults. Clin Infect Dis. 2007;44 Supp1 2:S27-S72.

8. Unkeless JC. Function and heterogeneity of human Fc receptors for immunoglobulin G. J Clin Invest. 1989;83(2):355-361.

9. Nuutila J. The novel applications of the quantitative analysis of neutrophil cell surface FcgammaRI (CD64) to the diagnosis of infectious and inflammatory diseases. Curr Opin Infect Dis. 2010;23(3):268-274.

10. Icardi M, Erickson Y, Kilborn S, Stewart B, Grief B, Scharnweber G. CD64 index provides simple and predictive testing for detection and monitoring of sepsis and bacterial infection in hospital patients. J Clin Microbiol. 2009;47(12):3914-3919.

11. Hoffmann JJ. Neutrophil CD64: a diagnostic marker for infection and sepsis. Clin Chem Lab Med. 2009;47(8):903-916.

12. Dellinger RP, Levy MM, Rhodes A, et al; Surviving Sepsis Campaign Guidelines Committee including The Pediatric Subgroup. Surviving Sepsis Campaign: international guidelines for management of severe sepsis and septic shock, 2012. Intensive Care Med. 2013;39(2): 165-228.

13. Pierrakos C, Vincent JL. Sepsis biomarkers: a review. Crit Care. 2010;14(1):R15.

14. Cardelli P, Ferraironi M, Amodeo R, et al. Evaluation of neutrophil CD64 expression and procalcitonin as useful markers in early diagnosis of sepsis. Int J Immunopathol Pharmacol. 2008;21(1):43-49.

15. Groselj-Grenc M, Ihan A, Pavcnik-Arnol M, Kopitar AN, GmeinerStopar T, Derganc M. Neutrophil and monocyte CD64 indexes, lipopolysaccharide-binding protein, procalcitonin and $\mathrm{C}$-reactive protein in sepsis of critically ill neonates and children. Intensive Care Med. 2009;35(11):1950-1958.

16. Davis BH, Olsen SH, Ahmad E, Bigelow NC. Neutrophil CD64 is an improved indicator of infection or sepsis in emergency department patients. Arch Pathol Lab Med. 2006;130(5):654-661. 
17. Gámez-Díaz LY, Enriquez LE, Matute JD, et al. Diagnostic accuracy of HMGB-1, sTREM-1, and CD64 as markers of sepsis in patients recently admitted to the emergency department. Acad Emerg Med. 2011;18(8): 807-815.

18. Horan TC, Andrus M, Dudeck MA. CDC/NHSN surveillance definition of health care-associated infection and criteria for specific types of infections in the acute care setting. Am J Infect Control. 2008;36(5): 309-332.

19. von Elm E, Altman DG, Egger M, Pocock SJ, Gøtzsche PC, Vandenbroucke JP; STROBE Initiative. The Strengthening the Reporting of Observational Studies in Epidemiology (STROBE) statement: guidelines for reporting observational studies. J Clin Epidemiol. 2008;61(4):344-349.

20. Trillium Diagnostics, LLC. Leuko64 ${ }^{\mathrm{TM}}$ Assay Kit. Brewer, ME: Trillium Diagnostics, LLC; 2012. Available from: http:/www.trilliumdx.com/ files/docs/131_leuko64_iuo_package_insert.pdf. Accessed April 19, 2014.

21. Ellis PD. The Essential Guide to Effect Sizes: Statistical Power, MetaAnalysis, and the Interpretation of Research Results. New York, NY: Cambridge University Press; 2010.

22. Christ-Crain M, Müller B. Biomarkers in respiratory tract infections: diagnostic guides to antibiotic prescription, prognostic markers and mediators. Eur Respir J. 2007;30(3):556-573.

23. Elghetany MT, Davis BH. Impact of preanalytical variables on granulocytic surface antigen expression: a review. Cytometry B Clin Cytom. 2005;65(1):1-5.

24. Allen E, Bakke AC, Purtzer MZ, Deodhar A. Neutrophil CD64 expression: distinguishing acute inflammatory autoimmune disease from systemic infections. Ann Rheum Dis. 2002;61(6):522-525.
25. Schuetz P, Amin DN, Greenwald JL. Role of procalcitonin in managing adult patients with respiratory tract infections. Chest. 2012;141(4): 1063-1073.

26. Porzecanski I, Bowton DL. Diagnosis and treatment of ventilatorassociated pneumonia. Chest. 2006;130(2):597-604.

27. Torres A, Ewig S, Lode H, Carlet J; European HAP working group. Defining, treating and preventing hospital acquired pneumonia: European perspective. Intensive Care Med. 2009;35(1):9-29.

28. Pauwels RA, Buist AS, Calverley PM, Jenkins CR, Hurd SS; GOLD Scientific Committee. Global strategy for the diagnosis, management, and prevention of chronic obstructive pulmonary disease. NHLBI/ WHO Global Initiative for Chronic Obstructive Lung Disease (GOLD) Workshop summary. Am J Respir Crit Care Med. 2001;163(5): 1256-1276.

29. Livaditi O, Kotanidou A, Psarra A, et al. Neutrophil CD64 expression and serum IL-8: sensitive early markers of severity and outcome in sepsis. Cytokine. 2006;36(5-6):283-290.

30. Gros A, Roussel M, Sauvadet E, et al. The sensitivity of neutrophil CD64 expression as a biomarker of bacterial infection is low in critically ill patients. Intensive Care Med. 2012;38(3):445-452.

31. Chan YL, Tseng CP, Tsay PK, Chang SS, Chiu TF, Chen JC. Procalcitonin as a marker of bacterial infection in the emergency department: an observational study. Crit Care. 2004;8(1):R12-R20.

32. Schuetz P, Chiappa V, Briel M, Greenwald JL. Procalcitonin algorithms for antibiotic therapy decisions: a systematic review of randomized controlled trials and recommendations for clinical algorithms. Arch Intern Med. 2011;171(15):1322-1331.
Open Access Emergency Medicine

\section{Publish your work in this journal}

Open Access Emergency Medicine is an international, peer-reviewed, open access journal publishing original research, reports, editorials, reviews and commentaries on all aspects of emergency medicine. The manuscript management system is completely online and includes a very quick and fair peer-review system, which is all easy to use.

\section{Dovepress}

Visit http://www.dovepress.com/testimonials.php to read real quotes from published authors. 